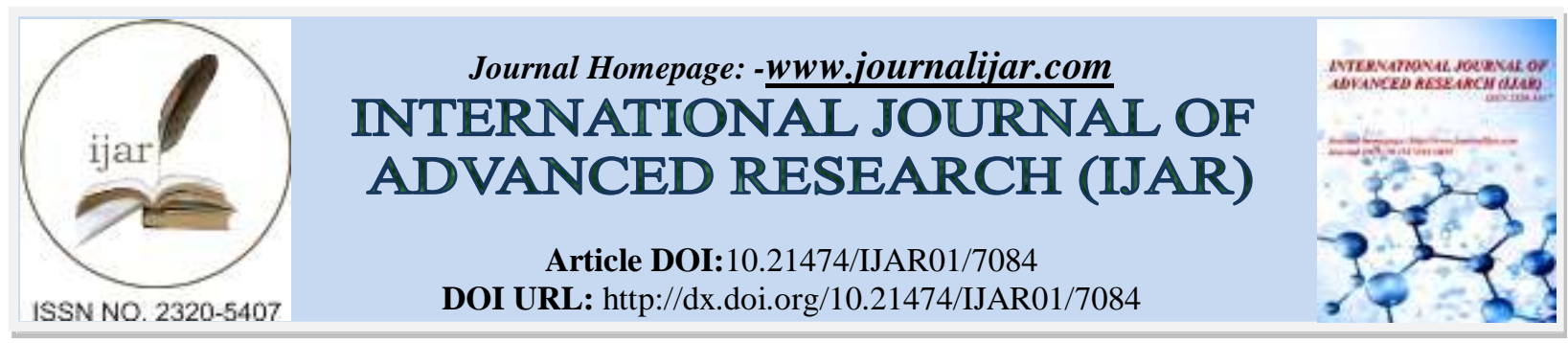

RESEARCH ARTICLE

\title{
IMPACTED KNIFE INJURY MAXILLOFACIAL REGION - A DIFFICUILT SCENARIO.
}

Dr. Sreekumar Ramachandran and Dr. Jayan Stephen.

1. Associate professor of Surgery, Govt. Medical College Hospital, Thiruvananthapuram, Kerala.

2. Assistant Professor of Surgery, Govt. Medical College Hospital, Thiruvananthapuram, Kerala.

\section{Manuscript Info}

Manuscript History

Received: 12 March 2018

Final Accepted: 14 April 2018

Published: May 2018

Keywords:-

Impacted knife, Maxillofacial region

,foregn bodies in the face, impacted

injury face, Neurovascular stability.

\section{Abstract}

Impacted foreign body face with knife in situ is an uncommon presentation. The proximity to vital structures and absence of strict management protocol often gives challenges to the consultant and his entire team. The neurovascular stability of the victim is the determinant for definitive investigations and planning of management protocol. Xray, CT scan and arteriogram were the main investigative tools. Here we are reporting successful removal of an impacted foreign body face, were $12 \mathrm{~cm}$ of the knife was inside.

Copy Right, IJAR, 2018,. All rights reserved.

\section{Introduction:-}

A variety of foreign bodies can be encountered in the maxillary antrum. These can be classified into: Traumaticmetal pieces, glass pieces, airgun pellets, stones, wood etc.; and Iatrogenic - dental cement, roots of teeth, whole tooth, broken pieces of hypodermic needles, impression paste, broken end of forceps.

\section{Case Report:-}

36 yr old lady was admitted to the causality with stab injury face (Figure. 1). She was stabbed by husband after alcohol intoxication. Long knife was used for the attack and it has completely penetrated into the face just below the right eye. The weapon was a $15 \mathrm{~cm}$ long knife using for agricultural purpose and was penetrated completely into the face on the right side. The entry wound was $2 \mathrm{cms}$ below the right eye in the middle. External eye was intact. Knife was not seen in the oral or nasal cavity. Vision was normal. No bleeding from mouth, nose or ear. Her sensorium was normal and no neurovascular deficits were there.No active bleeding present from the wound.Xray revealed the stab vertically into the face, penetrated the maxilla and reached up to the cervical spine (Figure. 2). CT head showed the trajectory of the foreign body and the tip was very close to the jugular bulb. Since there is no active bleeding, arteriogram was not done. (Figure.3). This paper delineates a rare case of impacted foreign body in the face, the importance of proper planning to treat such cases and our method of successful management.

\section{Management:-}

Case was discussed with anesthesiologist, neurosurgeon and otolaryngologists regarding line of management and possible complications. Her blood group was B negative and only one unit was available in the blood bank and since it was midnight there was practical difficulties to mobilize more. Patient was taken to operation theatre. GA was induced and the foreign body did not affect intubation. Anticipating the possible injury to the carotids and jugular vessels, we decided to explore the neck. Neck was opened through an oblique incision along the anterior border of the sternomastoid and carotid sheath was opened. Internal and external carotids and internal jugular vein were taped 
for control (Figure. 4). Jugular vein was traced upwards till we could see the tip of the knife. It was abutting with the venous wall .Under direct vision and care, knife was pulled out slowly. It was $15 \mathrm{cms}$ long of which $12 \mathrm{~cm}$ was inside the face (figure. 5 and 6). After removal, we could ensure that the vein is intact but there is significant arterial bleed. We could not locate the bleeding artery but on compressing the external carotid it stopped. So we have decided to ligate the external carotid, which led to hemostasis. The entry wound was washed with saline many times till everything is clear. Wound is closed in layers with drain.

Tetanus prophylaxis, ceftriaxone, metronidazole, analgesics and IV fluids were started and patient was transferred to ICU. Oral feed was started after 6 hours and she had a smooth recovery. No neurological or visual defects were noticed in the postoperative phase.

\section{Discussion:-}

Impacted foreign bodies of the face are very rare and very few series is available. The common causes are due to increasing violence as a result of easy availability of firearms, gang wars and warfare (battlefield) injuries, escape of teeth and dental impression materials through oro-antral fistula, Iatrogenic causes due to root filling procedures. No definite protocols are available on its management. We couldn't identify any report on prior exploration of neck in anticipating secondary injuries. Most of the foreign bodies can be pulled out without any significant damage. Regarding pre operative evaluation X-ray, CT scan and arteriogram are the important tools and arteriogram is indicated only in patients with active bleed. The usefulness of CT scan may be limited due to a large degree of scatter by the metallic object ${ }^{4}$, although it may prove useful when there is bony/soft tissue injury. Prior planning and multidisciplinary approach are the cornerstone of the successful management. Impacted foreign body should not be pulled immediately. As doing so may further damage surrounding vital tissues, particularly large blood vessels and nerves, which can be disastrous. It is best to leave the foreign body in situ and attempts for its removal should be made in the Operating room with all necessary equipment to manage complications 5 . The entry wound should be widened and explored to ensure the integrity of major blood vessels and vital structures. The wound should be thoroughly irrigated with saline and diluted povidone-Iodine solution. Post-operative complications are very few and the wound usually heals well.

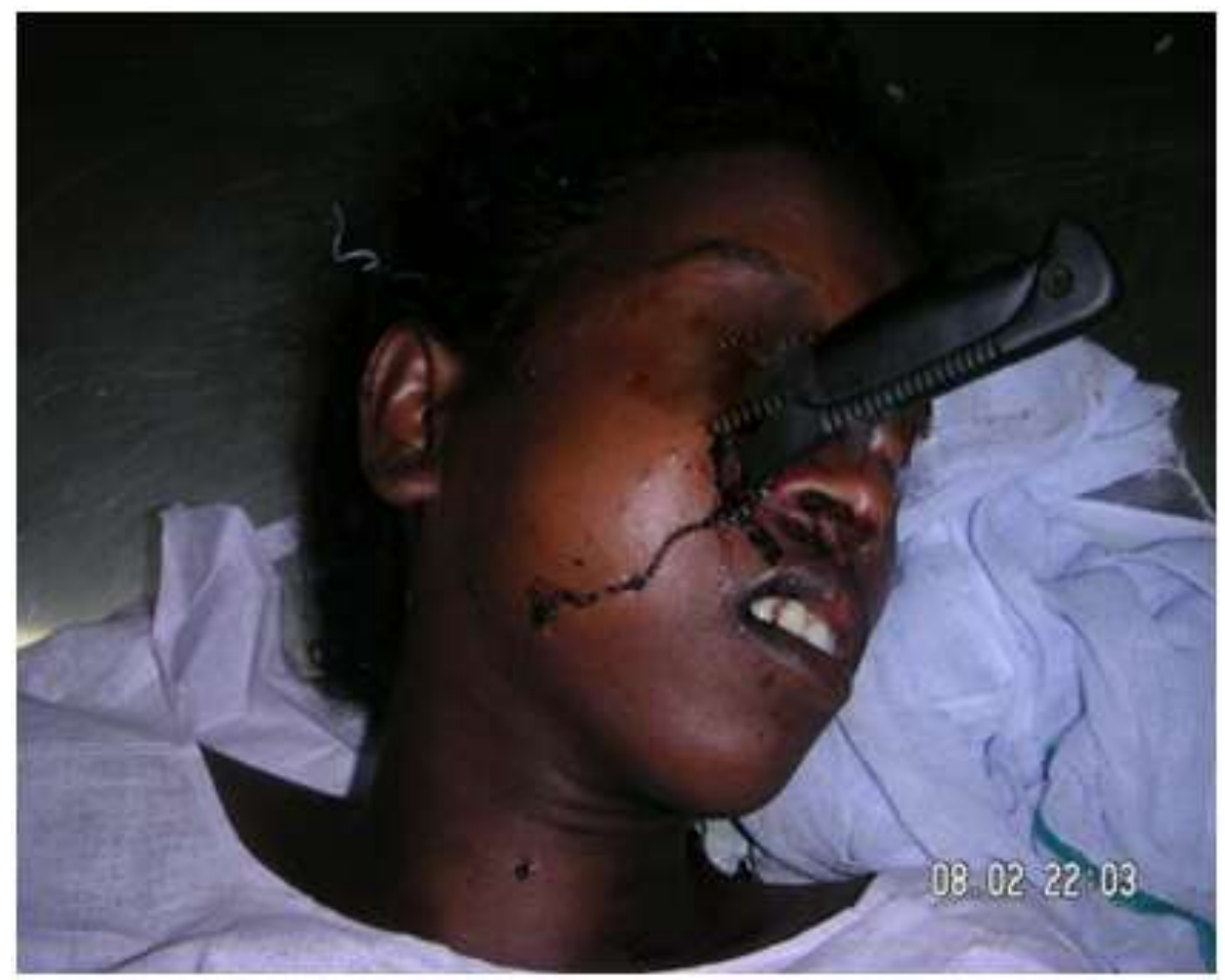

Figure 1:- showing knife insitu. 


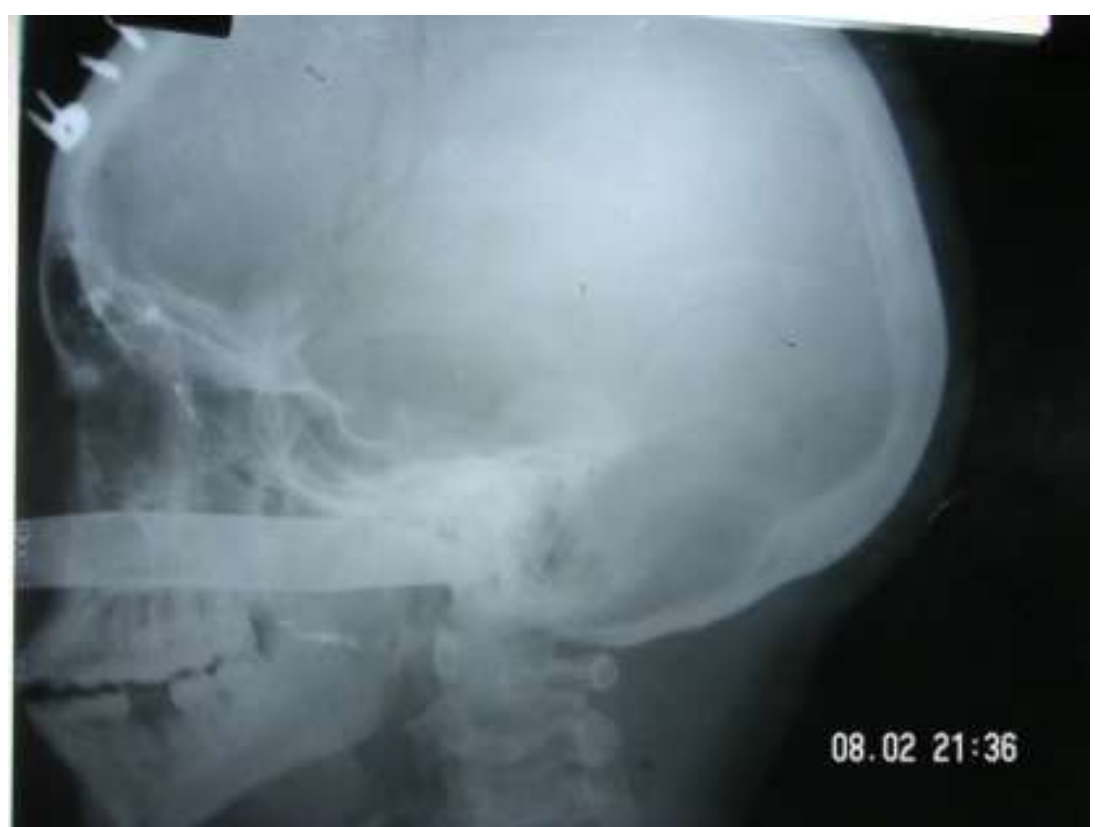

Figure 2:- $X$ ray showing tip of knife reached up to cervical spine

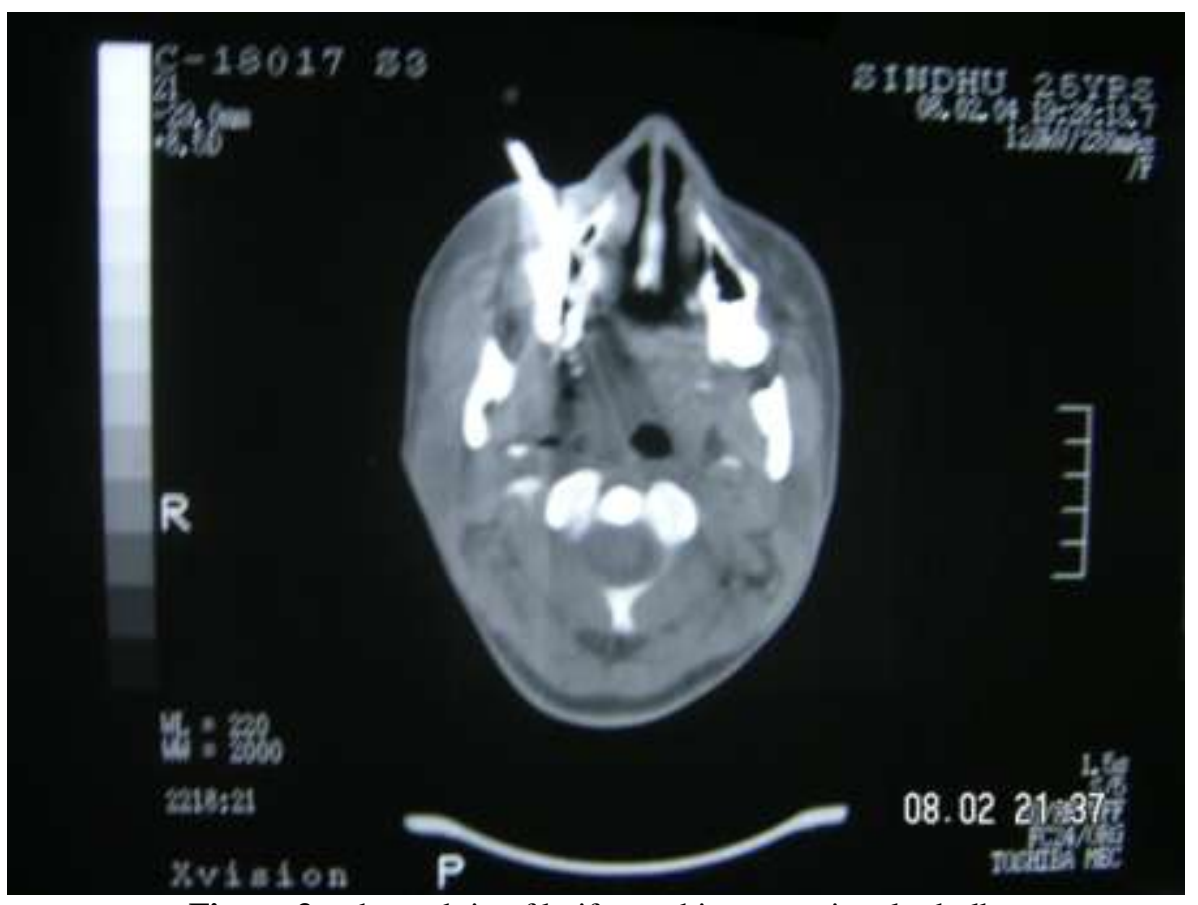

Figure 3:- showed tip of knife reaching up to jugular bulb 


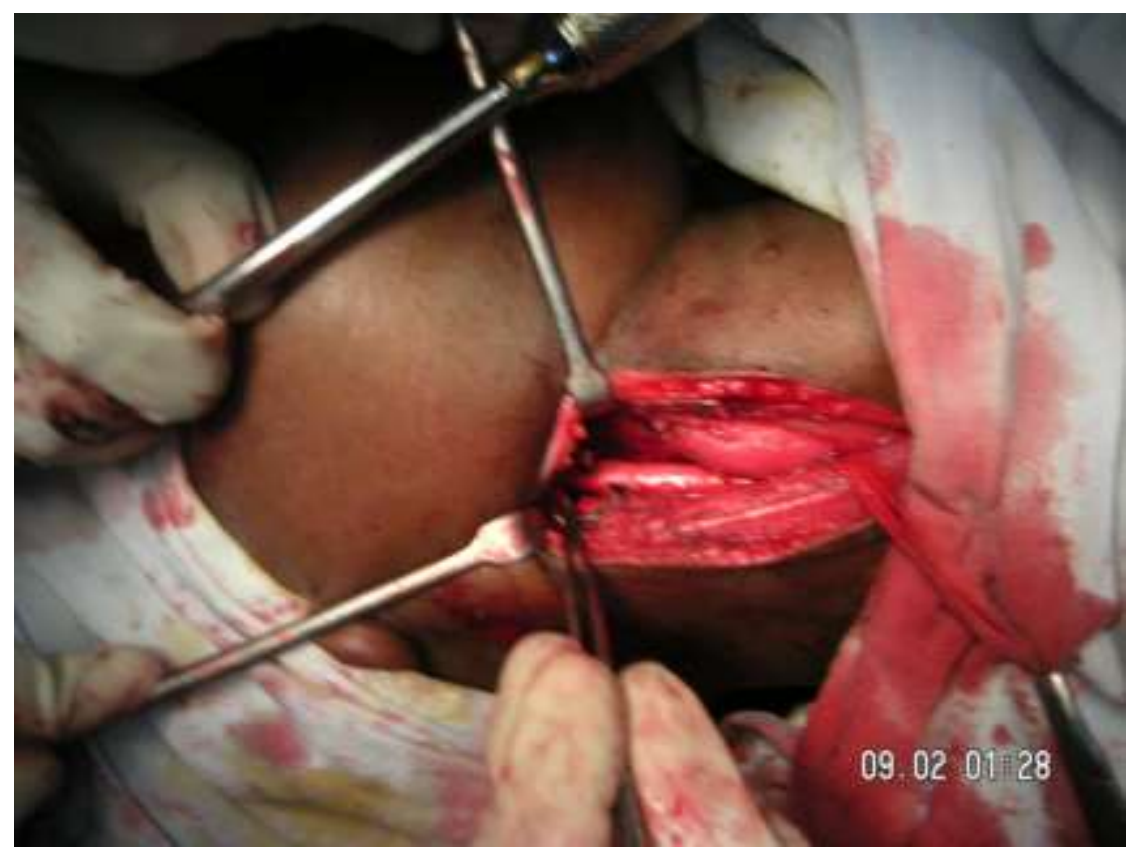

Figure 4:- Showing exposed carotid vessels

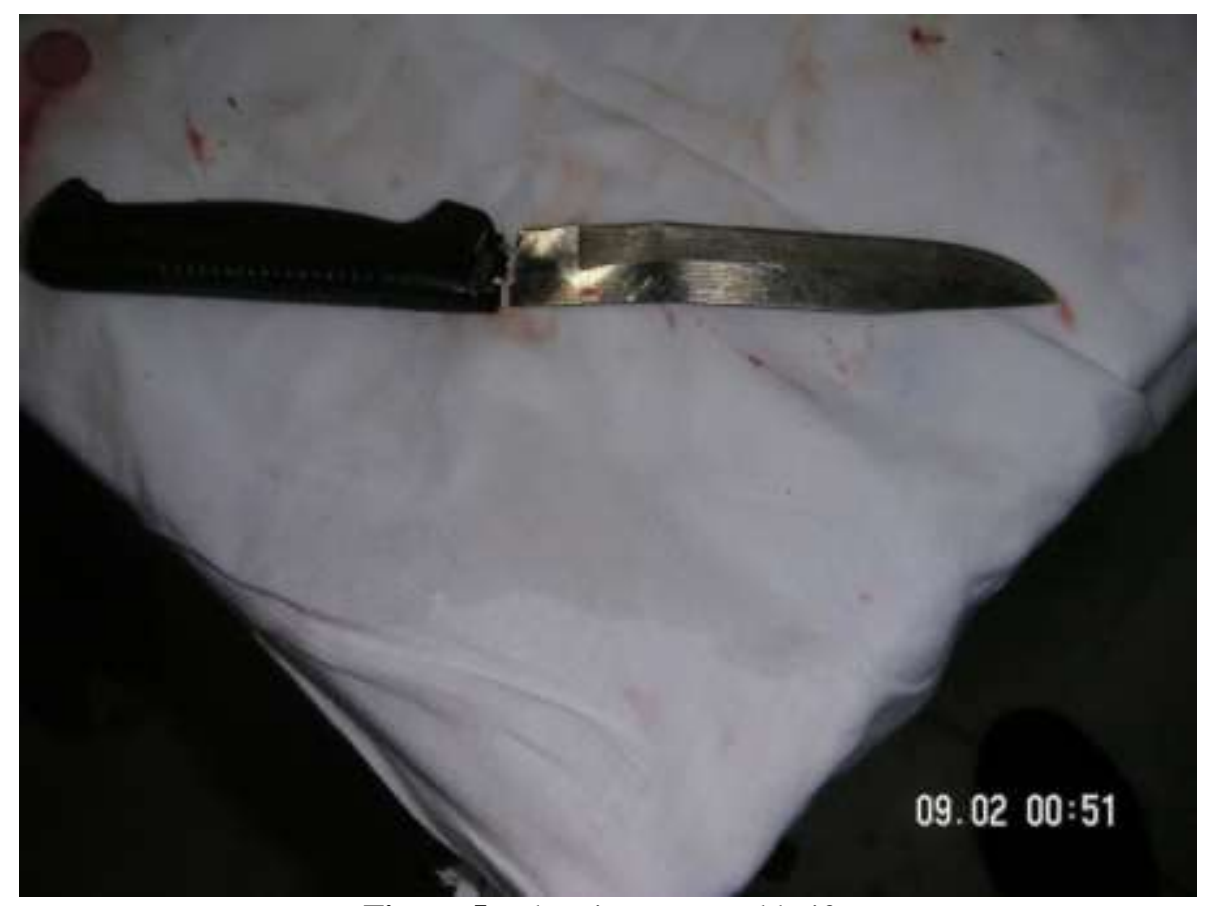

Figure 5:- showing removed knife 


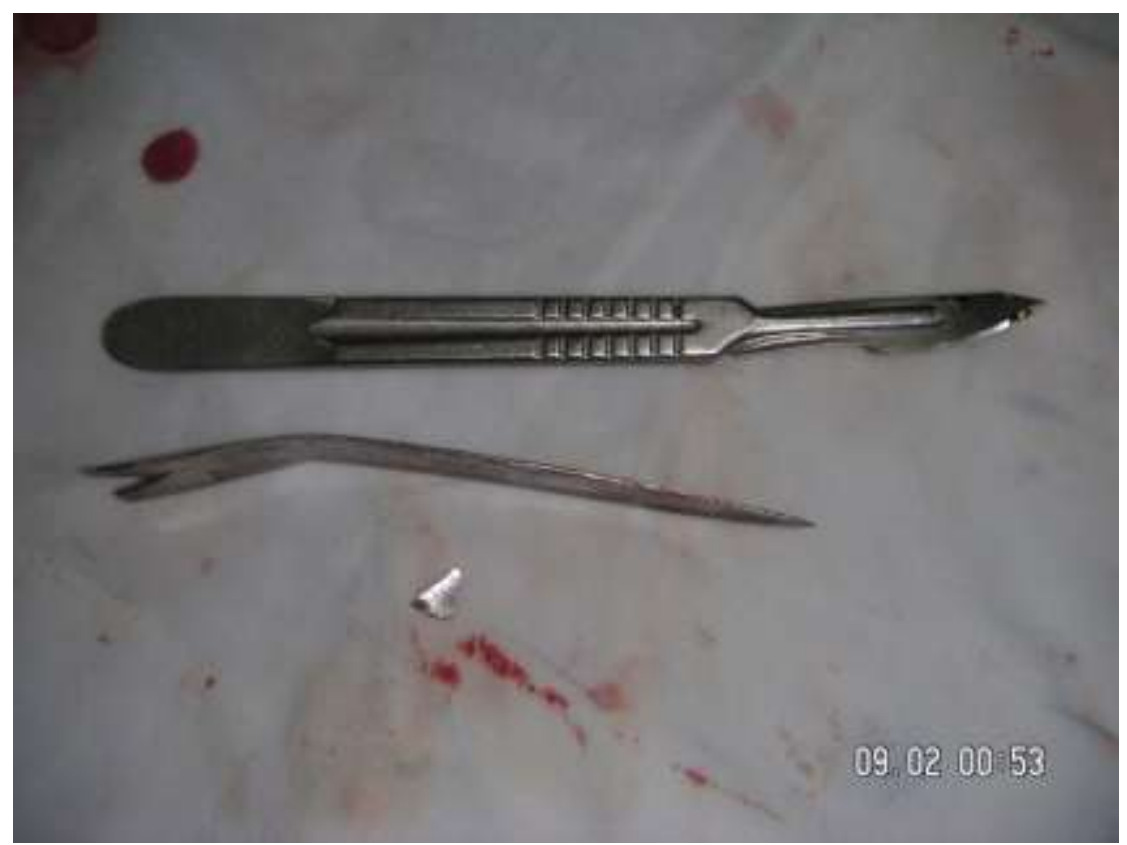

Figure 6:- showing length of impacted part of knife

\section{References:-}

1. Westermark AH. Spontaneous removal of foreign bodies from the maxillary sinus: Report of a case. Journal of Oral Maxillofacial Surgery. 1989;47:75- 77.

2. Gupta AC, Murthy DP, Pulotu ML. Unusual type of foreign body in the maxillary sinus. The Journal of Laryngology and Otology. 1990;104: 718-9.

3. Sandu KB, ShahNJ, Kirtane MV. Foreign body in the maxillary antrum. A case report.Int J Oral Maxillofac Surgery. 1997;26:110-1.

4. O'Connel JE, Turner NO, Pahor AL. Air gun pellets in the sinuses. The Journal of Laryngology and Otology. 1995;109:1097-1100. 